\title{
Nuevos datos sobre la biología y distribución de la especie (Dyscinetus dubius) en Colombia, descripción de larva y pupa y clave para la identificación de las larvas del tercer estadio de las especies conocidas para el género Dyscinetus
}

\section{New data on the biology and distribution of the species (Dyscinetus dubius) in Colombia, description of larva and pupa and key for the identification of the larvae of the third stage of the species known for Dyscinetus genus}

\begin{abstract}
Jhon César Neita-Moreno, MSc ${ }^{1}$, Francisco Yepes, MSc $^{2}$
\end{abstract}
1. Grupo de Sistemática de Insectos Agronomía, Museo Entomológico Universidad Nacional Agronomía Bogotá (UNAB), Facultad de Agronomía, Universidad Nacional de Colombia, Bogotá. Investigador Asociado, Instituto de Investigaciones Ambientales del Pacífico (IIAP), Quibdó, Colombia.e-mail: cneitaj@unal.edu.co

2. Profesor, Facultad de Ciencias Agropecuarias, Departamento de Ciencias Agronómicas, Universidad Nacional de Colombia, Sede Medellín, Colombia. e-mail: fcyepes@unalmed.edu.co Recibido: 21 de febrero de 2011 Aceptado: 19 de abril de 2011

Melolonthidae: Dynastinae: Cyclocephalini) are described. New data about the biology and distribution of this species in Colombia are reported and a key to the known third stage larvae of the species of Dyscinetus is provided.

Keywords: Taxonomic key; Scarabs; Natural history; Immature.

\section{INTRODUCCIÓN}

El género Dyscinetus contiene 15 especies que se distribuyen desde el centro de los Estados Unidos a Argentina (Endrödi 1966, 1985; Ratcliffe 2003). En Colombia se han registrado seis especies (Restrepo et al. 2003). Las larvas de Dyscinetus Harold (Ritcher 1966; Vincini et al. 2000), se diferencian de otros géneros en Cyclocephalini y Dynastinae por la siguiente combinación de caracteres: sedas frontales posteriores y externas presentes, sedas anteriores ausentes. Ocelos presentes, no pigmentados. Haptómero entero. Bordes anteriores de la epifaringe angulados. Epifaringe con algunas sensilas entre las sedas. Laeotorma dilatada apicalmente. Lacinia con tres unci fusionados en la base; área estriduladora maxilar con siete dientes truncados y proceso ventral anterior presente. Área incisiva de la mandíbula izquierda con el cuarto diente presente. Dos áreas sensoriales dorsales en el último artejo antenal. Palidia ausente. Abertura anal ligeramente curvada. El presente trabajo tiene como objetivo presentar nuevos datos sobre la biología y distribución en Colombia de Dyscinetus dubius (Olivier 1789) (Coleoptera: Melolonthidae: Dynastinae: Cyclocephalini), describir los estados inmaduros de la especie y elaborar una clave para la identificación de las larvas de tercer estadio del género Dyscinetus.

\section{MATERIALES Y MÉTODOS}

Las larvas descritas en el presente artículo fueron capturadas durante la investigación «Taxonomía y distribución de chisas (Coleoptera: Melolonthidae) asociadas a cinco cultivos de importancia agrícola en Cundinamarca, Colombia» entre los años 2003-2006. El 50\% de los especímenes de los estados inmaduros obtenidos en el campo se fijaron en solución de Pampel (Stehr 1987) y el otro 50\% se utilizó para obtener exuvias larvales, pupas y adultos en el laboratorio y así corroborar la identidad taxonómica.

Cada larva se depositó en un recipiente de $200 \mathrm{ml}$ con tierra 


\section{Bioetnia Volumen 8 No 1 (enero-junio), 2011}

y plántulas de trigo para que culminara su desarrollo. Para ello se dispuso de un cuarto con temperatura controlada acorde con las condiciones de humedad ( $78 \%$ a $90 \%$ ) y temperatura $\left(28^{\circ} \mathrm{C}\right)$ del hábitat natural de las larvas. Esta actividad se realizó durante 12 meses.

De las muestras se tomaron seis larvas de tercer estadio para realizar las descripciones e ilustraciones y 14 ejemplares para obtener las mediciones de cápsula cefálica y longitud del cuerpo. Las observaciones, ediciones e ilustraciones se realizaron con la ayuda del estereoscopio. Los caracteres y la terminología utilizada en las descripciones se basan en las propuestas por Böving (1936, 1942); Ritcher (1966) y Morón (1987, 1996). Los especímenes tanto larvas como adultos se depositaron en la colección de formas inmaduras del Museo Entomológico de la Universidad Nacional Agronomía Bogotá (UNAB).

\section{RESULTADOS}

\section{Descripción de larva y pupa}

Larva de tercer estadio. Cabeza (Figura 1): Amplitud de la cápsula cefálica: 4,6-4,7 mm. Superficie del cráneo ligeramente rugosa de color pardo oscuro. Frente con 3-4 sedas en los ángulos frontales anteriores, dos sedas dorsoepicraneales, 16 sedas epicraneales irregularmente distribuidas y 8-10 sedas paraocelares a cada lado. Una seda frontal posterior y una seda frontal exterior. Sedas frontales anteriores ausentes. Ocelos pequeños, bien definidos. Clípeo con dos sedas centrales y dos sedas laterales a cada lado. Labro: Ligeramente asimétrico con cuatro sedas laterales, dos sedas centrales y cuatro sedas posteriores. Epifaringe (Figura 2) 1,4 mm de ancho por $0,9 \mathrm{~mm}$ de largo. Borde lateral posterior izquierdo angulado. Proceso haptomeral prominente y entero. Acanthoparia con 11-14 sedas espiniformes cortas recurvadas. Corypha con cinco sedas delgadas y cortas. Acroparia con 29 sedas delgadas. Dexiotorma delgadas y extremo mesal aguzado. Laeotorma más corta que la dexiotorma; extremo mesal dilatado proyectándose hacia el pedium, con pterotorma redondeada y pronunciada. Placa esclerosada grande y angulosa; cono sensorial ancho y prominente con cuatro sensilias. Laeophoba consistente en 18 sedas delgadas y cortas ligeramente esparcidas entre el haptolechus y la laeotorma. Crepis fino, ligeramente esclerosado y bien definido. Pedium amplio. Chaetoparia izquierda con 60-62 sedas. Chaetoparia derecha con 66-68 sedas. Mandíbula derecha (Figuras 4a-4b): Forma falcada. Área incisiva con tres dientes ( 1 y $\mathrm{S} 2$ ) fusionado y S3 después de la escotadura incisiva. Scrobis con cinco sedas delgadas y largas. Superficie dorsal con una línea de ocho sedas dorsomolares. Mandíbula izquierda (Figuras 3a-3b). Área incisiva con tres dientes S1+S2 fusionados, S3 separado por la escotadura postincisiva y $\mathrm{S} 4$ truncado, scrobis con cinco sedas largas y delgadas. Super- ficie dorsal con una línea de seis sedas dorsomolares; bien desarrollada, alargada y triangular, con tres sedas apicales cortas y delgadas. Superficie ventral con un área estriduladora ovalada con 30 líneas estriduladoras; proceso ventral desarrollado, brustia con nueve sedas moderadamente larga y gruesa dispuesta en forma de «U» y sedas basolaterales ausentes. Área molar con tres lóbulos, el primero más desarrollado que el segundo y el tercero.

Superficie ventral con área estriduladora ovalada con 30 líneas; proceso ventral desarrollado; brustia con cuatro sedas moderadamente largas y gruesas. Calx prominente; sedas basolaterales ausentes. Área molar con tres lóbulos, el primero más desarrollado. Maxila (Figuras 5a-5b): cardo subtriangular. Estipe más largo que ancho. Galea con muchas sedas gruesas y un uncus bien desarrollado, rodeado por seis heli gruesos. Lacinia con muchas sedas gruesas, delgadas, mezcladas y tres unci unidos en la base, rodeados por ocho heli gruesos. Palpos de cuatro artejos. Área estriduladora maxilar (Figura 6) con siete dientes truncados y un proceso anterior. Labio (Figura 7). Superficie de la glosa con 47 sedas delgadas y largas laterales; 36 sedas gruesas y cortas en el centro. Escleroma hipofaríngeo asimétrico, cóncavo mesalmente y un proceso dorsal en el lado derecho. Lóbulo lateral derecho con 13 sedas cortas y delgadas. Lóbulo lateral izquierdo con once sedas delgadas y moderadamente largas, una línea de 22 sedas moderadamente largas y gruesas rodean el escleroma con dirección hacia el centro; tres sedas gruesas en la base del escleroma. Palpos labiales con dos artejos, el segundo dos veces más largo que el primero. Antena. Compuesta de cuatro artejos. Artejo apical con dos áreas sensoriales dorsales (Figura 8) y dos áreas sensoriales ventrales (Figura 9).

Tórax. Pronoto con un escleroma lateral de bordes obtusos, con seis sedas delgadas y largas. Los estigmas respiratorios ( $0,37 \mathrm{~mm}$ de largo por 0,23 $\mathrm{mm}$ de ancho) en forma de «C» (Figura 10a), la distancia entre los lóbulos de la placa respiratoria es menor que el diámetro dorso-ventral de la bulla poco prominente; con 27 perforaciones en promedio en el radio de la placa respiratoria (Figura 10b), placa respiratoria con bordes definidos. Dorso del protórax con una línea de ocho sedas delgadas y largas; prescudo mesotorácico con una línea irregular de seis sedas delgadas y largas; escutelo mesotorácico con seis sedas delgadas y largas; prescudo metatorácico con cuatro sedas largas, delgadas y con seis sedas cortas espiniformes; escudo metatorácico con 16 sedas delgadas, largas y 17 sedas cortas espiniformes; escutelo metatorácico con 12 sedas delgadas, largas y cuatro sedas cortas espiniformes. Patas. Pro y metatarsungulos más pequeños que los mesotarsungulos, alargados con el ápice agudo, con una seda basal interna y una seda prebasal externa (Figuras 11a-11b).

Abdomen. Estigmas del primer segmento abdominal más 

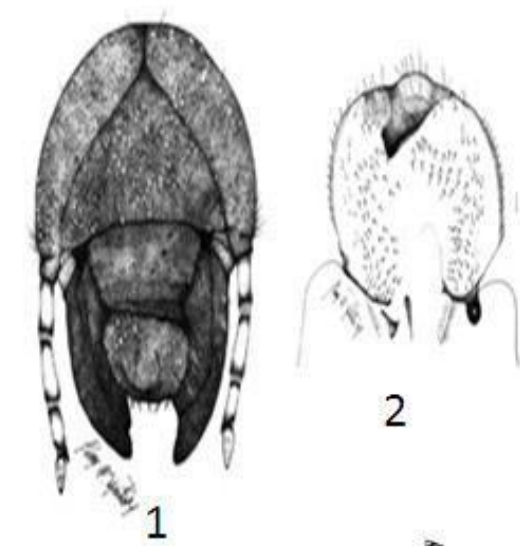

2

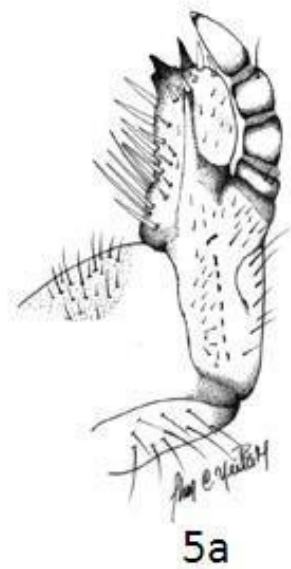

$4 b$

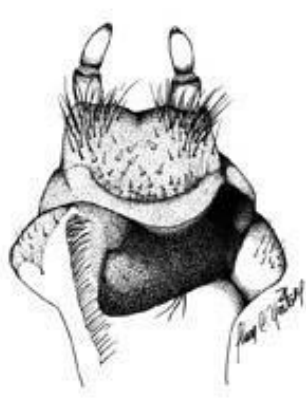

7

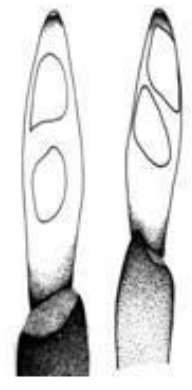

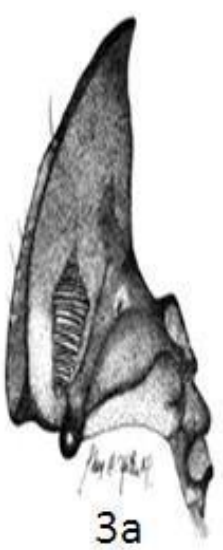
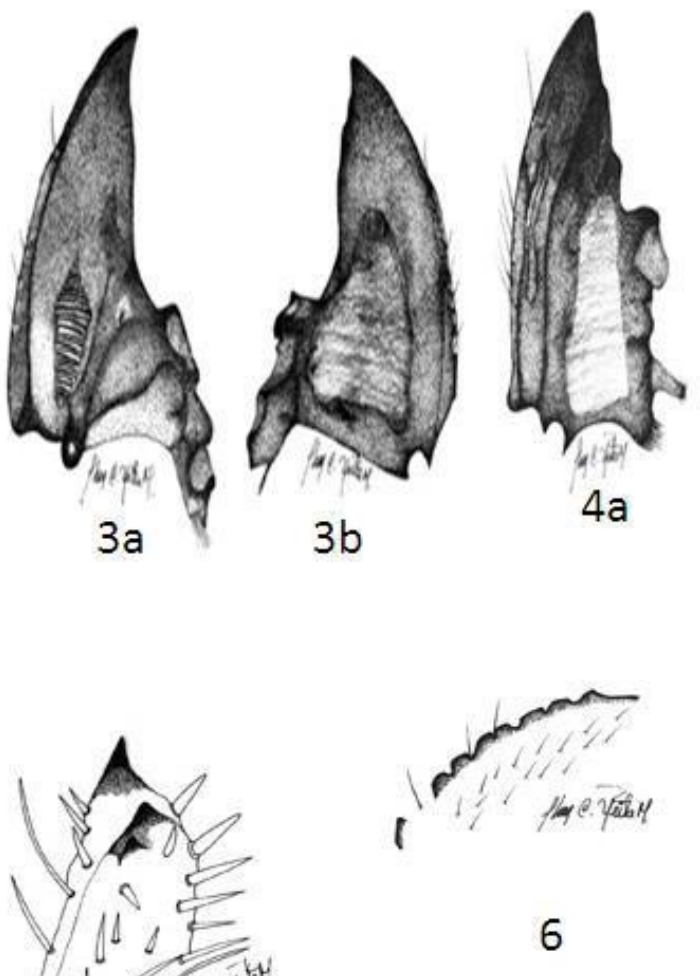

6

$5 b$
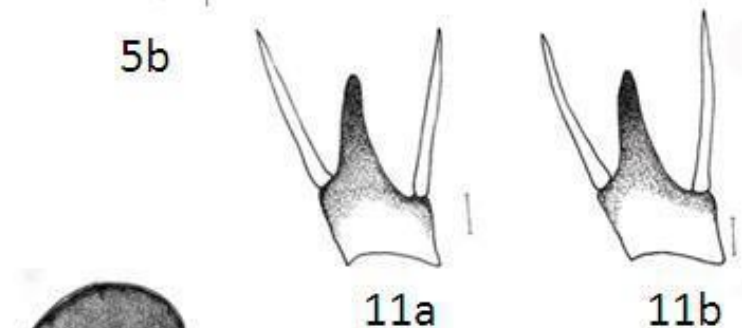

$11 \mathrm{~b}$

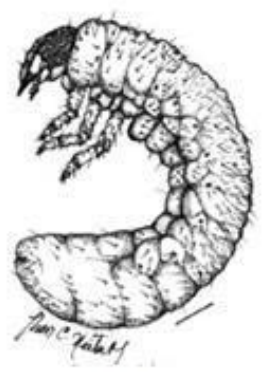

12

13

Figura 1. Cabeza vista frontal. Figura 2. Epifaringe. Figura 3. Mandíbula derecha. Figura 3a. Vista dorsal; Figura 3b. Vista ventral. Figura 4. Mandíbula izquierda. Figura 4a. Vista dorsal; Figura 4b. Vista ventral; Figura 5. Maxila. Figura 5a. Vista ventral; Figura 5b. Vista interna. Figura 6. Dientes estriduladores de la maxila; Figura 7. Labio hipofaríngeo; Figura 8. Superficie dorsal del último artejo antenal. Figura 9. Superficie ventral del último artejo antenal; Figura 10a. Espiráculo protorácico; Figura 10b. Perforaciones de la placa respiratoria. Figura 11a. Mesotarsungulo; Figura 11b. Metatarsungulo. Figura 12. Larva de tercer estadio. Figura 13. Vista lateral de la pupa macho. 


\section{Bioetnia Volumen 8 No 1 (enero-junio), 2011}

pequeños (0,32 $\mathrm{mm}$ de largo por $0,2 \mathrm{~mm}$ de ancho) que los estigmas de los II-VIII; estigmas de los segmentos II-VI ligeramente más pequeños $(0,32 \mathrm{~mm}$ de largo por $0,25 \mathrm{~mm}$ de ancho) que los estigmas VII y VIII ( $0,32 \mathrm{~mm}$ de largo por 0,28 $\mathrm{mm}$ de ancho); placas respiratorias de color marrón oscuro, regularmente recurvada en forma de $« \mathrm{C} »$, la distancia entre los lóbulos de las placas respiratorias es menor que el diámetro dorso-ventral de la bulla poco prominente. Área espiracular con nueve sedas delgadas y largas. Segmento abdominal I, prescudo con 18 sedas delgadas, largas y dos sedas cortas espiniformes; subescudo sin sedas; escudo con seis sedas delgadas, largas y 46 sedas cortas espiniformes; escutelo con ocho sedas delgadas, largas y 38 sedas cortas espiniformes. Área espiracular con nueve sedas delgadas y largas. Segmento abdominal II, prescudo con cuatro sedas delgadas, largas y 29 sedas cortas, espiniformes; subescudo con cuatro sedas largas y delgadas; escudo con diez sedas delgadas, largas y 75 sedas cortas espiniformes; escutelo con ocho sedas delgadas, largas y 52 sedas cortas espiniformes. Área espiracular con nueve sedas delgadas y largas. Segmento abdominal III, prescudo con cuatro sedas delgadas, largas y 40 sedas cortas, espiniformes; subescudo con cuatro sedas largas y delgadas; escudo con cuatro sedas delgadas, largas y 40 sedas cortas espiniformes; escutelo con ocho sedas delgadas, largas y 54 sedas cortas espiniformes. Área espiracular con nueve sedas delgadas y largas. Segmento abdominal IV, prescudo con cuatro sedas delgadas, largas y 53 sedas cortas espiniformes; subescutum con cuatro sedas largas y delgadas; escudo con ocho sedas delgadas, largas y 73 sedas cortas, espiniformes; escutelo con ocho sedas delgadas, largas y 57 sedas cortas espiniformes. Área espiracular con nueve sedas delgadas y largas. Segmento abdominal V, prescudo con cuatro sedas delgadas, largas y 32 sedas cortas, espiniformes; subescudo con cuatro sedas largas y delgadas; escudo con diez sedas delgadas, largas y 69 sedas cortas espiniformes; escutelo con ocho sedas delgadas, largas y 57 sedas cortas espiniformes. Área espiracular con nueve sedas delgadas y largas. Segmento abdominal VI, prescudo con dos sedas delgadas, largas y 78 sedas cortas espiniformes; subescudo con cuatro sedas largas y delgadas; escudo con 16 sedas delgadas, largas y 63 sedas cortas espiniformes; escutelo con cuatro sedas delgadas, largas y 47 sedas cortas espiniformes. Área espiracular con nueve sedas delgadas y largas. Segmento abdominal VIII con dos líneas transversales, línea anterior con ocho sedas delgadas y largas; línea posterior con ocho sedas delgadas, largas y seis sedas cortas espiniformes. Área espiracular con nueve sedas delgadas y largas. Segmento abdominal IX con una línea anterior de seis sedas largas y delgadas; línea posterior con diez sedas delgadas y largas. Sedas cortas ausentes. Segmento abdominal X con 158 sedas delgadas, largas y 142 sedas cortas espiniformes. Lóbulos pleurales con nueve sedas largas y delgadas, sedas cortas espiniformes ausentes. Raster: Sin palidia; campus con seis sedas delgadas y cortas; teges con 74 sedas hamate; barbula con 26-36 sedas delgadas y largas. Abertura anal transversa. Longitud de la larva $44 \mathrm{~mm}$ (Figura 12).

Pupa (Figura 13) (macho). 19,5 mm de largo por 8,5 mm de ancho. Cabeza. Con cobertura tomentosa fina, fuertemente inclinada hacia abajo; piezas bucales claramente diferenciadas, frente con superficie irregular. Clípeo convexo fusionado al labro; canthus ocular aparente; ojos hundidos, poco prominentes; tecae antenales engrosados, claramente visibles. Tórax. Pronoto convexo, ligeramente elevado hacia los bordes laterales; meso y metanoto bien diferenciados; tecae elitrales más cortas que las tecae alares; tecae elitrales se proyectan hasta la mitad del tercer segmento abdominal, tecae alares proyectándose hasta el cuarto segmento abdominal. Abdomen. Segmentos III-X (vista ventral) bien definidos; segmento VIII 0,25 veces más grande que el VII; segmentos IX y X fusionados. Segmentos I-X (vista dorsal) con órganos dioneiformes bien definidos entre los segmentos I-II, II-III, IIIIV, IV-V, V-VI, VI-VII. Lóbulos pleurales redondeados. Espiráculo I elongado con un peritrema fino y cubierto por el tecae alar; espiráculos de II-IV ovalado, prominentes con un peritrema fuertemente esclerosado; espiráculos V-VIII cerrados, espiráculos del segmentos VIII se proyectan con sentido mesial. Urogomphi ausentes, sin lóbulos laterales pleurales, ápices redondeados con cobertura tomentosa, doradas; ámpula genital ligeramente pronunciada en el macho.

Clave para la identificación de las larvas de tercer estadio del género Dyscinetus

1. Dorso del epicráneo con 4-7 sedas. Ángulo frontal anterior con una seda. Epifaringe con numerosas sensilas entre las sedas (Guatemala a Estados Unidos) ........... D. morator (Fabricius)

1'. Dorso del epicráneo con dos sedas. Ángulo frontal anterior con más de dos sedas. Epifaringe con pocas sensilas esparcidas entre las sedas

2

2(1'). Ángulo frontal anterior con 3-4 sedas. Laeophoba con 18 sedas. Labro con cuatro sedas laterales (México a Argentina) D.dubius(Olivier)

2'. Ángulo frontal anterior con dos sedas. Laeophoba con ocho sedas. Labro con tres sedas laterales (Brazil, Bolivia, Paragua y Argentina................. D. rugifrons (Burmeister)

Biología. Las larvas pupan a una profundidad de 25-38 $\mathrm{cm}$. Es posible capturar las pupas entre noviembre y finales de enero. Las pupas quedan retenidas en las exuvias larvales al igual que otros géneros de la tribu Cyclocephalini. Adultos. De hábitos nocturnos, son atraídos por las fuentes de luz. Vuelan entre las 7:00 p.m. y las 6:00 a.m. La emergencia de los adultos coincide con las primeras lluvias del año (marzojunio). Su ciclo de vida es anual. 
Distribución. Esta especie es conocida de México a Argentina (Endrödi 1966, 1985; Ratcliffe 2003) y su rango de distribución altitudinal abarca desde el nivel del mar a los 1700 $\mathrm{m}$. En Colombia esta especie se distribuye en los departamentos de Antioquia, Cauca, Córdoba, Chocó, Cundinamarca, Meta, Risaralda, Santander y Valle del Cauca.

Material examinado. 150 larvas de tercer estadio y 106 pupas (68@\& y 38 B\&). COLOMBIA. Cundinamarca. Fusagasugá. Vereda Jordán Bajo. Finca Santa Ana. $74^{\circ} 21^{\prime} \mathrm{W}$ $4^{\circ} 20^{\prime} \mathrm{N}$. $1731 \mathrm{~m}$. En kikuyo (Pennisetum clandestinum)Poaceae. 8-feb-2005. Neita, J. C. [UNAB]. COLOMBIA. Cundinamarca. San Francisco. Vereda Arrayanes. Finca Los Tibabuyes. $74^{\circ} 17^{\prime} \mathrm{W} 4^{\circ} 58^{\prime} \mathrm{N}, 1770 \mathrm{~m}$. En yuca (Manihot esculenta)-Euphorbiaceae. 14-mar-2005.Neita, J. C. [UNAB]. COLOMBIA. Cundinamarca. Manta. Vereda Las Palmas. $73^{\circ} 32^{\prime} \mathrm{W} 5^{\circ} 00^{\prime} \mathrm{N} .1924 \mathrm{~m}$. En yuca (Manihot esculenta)Euphorbiaceae. 4-abr-2005. Neita, J.C. [UNAB]. COLOMBIA. Cundinamarca. Gama. Finca Los Cerezos. $73^{\circ} 36^{\prime} \mathrm{W} 4^{\circ} 45^{\prime} \mathrm{N}$. $2180 \mathrm{~m}$. En yuca (Manihotesculenta)-Euphorbiaceae. 5-may2005. Neita, J.C. *UNAB+. COLOMBIA. Antioquia. Apartadó. Finca bananera. $7^{\circ} 53^{\prime} \mathrm{N} 76^{\circ} 40^{\prime} \mathrm{W} .30 \mathrm{~m}$. En suelo (Musa sp.)Musaceae. 2-ene-2004. González, J. C. [UNAB].

\section{AGRADECIMIENTOS}

El primer autor agradece a la Universidad Nacional de Colombia, sede Bogotá, en especial al Museo Entomológico UNAB por su apoyo logístico, a las familias que colaboraron durante el trabajo de campo en los municipios estudiados en
Cundinamarca. Este trabajo hace parte del proyecto Plagas Subterráneas de América del Sur (Centro Internacional de Agricultura Tropical (CIAT) y la Agencia Alemana AGZ.

\section{LITERATURA CITADA}

Böving, A. G. 1936. Description of the larva of Plectris aliena Chapin and explanation of new terms applied to the epipharynx and raster. Proc Entomol Soc Wash. 38: 169-85.

Böving, A. G. 1942. Descriptions of the larvae of some West Indian Melolonthinae beetles and a key to the known larvae of the tribe. Proc US Natl Mus. 92: 167-76.

Endrödi, S. 1966. Monographie der Dynastinae (Coleoptera, Lamellicornia). I. Teil. Ent Abh Mus Tierk. 33: 1-460.

Endrödi, S. 1985. The Dynastinae of the world. Dordrecht: Dr. W. Junk Publ. 800 pp. 46 plates.

Morón, M. A. 1987. Los estados inmaduros de Dynastes hyllus (Chevrolat) (Coleoptera: Melolonthidae: Dynastinae); con observaciones sobre biología y el crecimiento alométrico del imago. Folia Entomol Mexic. 72: 3374.

Morón, M. A. 1996. Melolonthidae (Coleoptera). p. 287-307. En: LlorenteBousquets, J. L., A. N. García-Aldrete, E. Gonzáles-Soriano (Eds). Biodiversidad, taxonomía y biogeografia de artrópodos de México. Hacia una sintesis de su conocimiento. México: CONABIO y UNAM. 660 pp.

Ratcliffe, B. C. 2003. The scarab beetles of Nebraska. Bull Univ Nebraska St Mus. 12: $1-333$

Restrepo, H., M. A. Morón, L. F. Vallejo, A. López, L. C. Pardo. 2003. Catálogo de Coleoptera: Melolonthidae (Scarabaeidae: Pleurosticti) en Colombia. Folia Entomol Mex. 42 (2): 239-63.

Ritcher, P. O. 1966. White Grubs and Their Allies. Oregon: Oregon State University Press. Corvallis. 219 pp.

Stehr, F. 1987. Immature insects I. Michigan: Department of Entomology, Michigan State University. 974 pp.

Vincini, A. M., A. N. López, P. L. Manetti, H. Álvarez-Castillo, D. M. Carmiona. 2000. Descripción de los estados inmaduros de Dyscinetus rugifrons (Burmeister, 1847) (Coleoptera: Scarabaeidae: Dynastinae). Elytron. 14: 91-8. 